$01,02,03$

\title{
Локализация носителей в квантовых точках с одноосной анизотропией формы и состава
}

\author{
(C) М.А. Семина, А.А. Головатенко, Т.В. Шубина, А.В. Родина \\ Физико-технический институт им. А.Ф. Иофрфе РАН, \\ Санкт-Петербург, Россия \\ E-mail: msemina@gmail.com
}

(Поступила в Редакцию 10 декабря 2018 г.

В окончательной редакции 10 декабря 2018 г.

Принята к публикации 11 декабря 2018 г.)

\begin{abstract}
Представлены результаты теоретического исследования электронных и дырочных состояний в квантовых точках (КТ) на основе кубических полупроводников II-VI сфероидальной формы, характеризующихся одноосной анизотропией. Рассмотрены гладкие потенциальные энергетические профили, моделируемые функцией Гаусса во всех трех пространственных направлениях. Проанализировано понижение уровня энергии, а также расщепление по энергии четырехкратно вырожденного состояния дырок из вершины валентной зоны $\Gamma_{8}$ с моментом $3 / 2$ на состояния с проекциями $\pm 3 / 2, \pm 1 / 2$ на ось анизотропии. Рассмотрена анизотропия КТ трех видов: анизотропия размера КТ, анизтропия потенциального барьера КТ и комбинированная анизотропия. В первом случае рассмотрены сплюснутые квантовые точки, характерный размер которых в плоскости структуры больше чем размер вдоль оси анизотропии. Во втором случае рассмотрены КТ, в которых высота потенциального барьера в плоскости меньше, чем высота потенциального барьера вдоль оси анизотропии. В третьем случае рассмотрены сплюснутые квантовые точки с анизотропией как размера так и потенциального барьера. Установлены условия локализакции носителей заряда внутри КТ и обсуждаются влияние анизотропии формы и состава на энергии экситонных переходов структур с квантовыми точками $\mathrm{Cd}_{x} \mathrm{Zn}_{1-x} \mathrm{Se}$.
\end{abstract}

Работа выполнена при поддержке Российского научного фонда (проект № 14-22-00107).

DOI: 10.21883/FTT.2019.04.47405.335

\section{1. Введение}

Твердотельные квантовые точки (КТ) считаются почти идеальными источниками квантового света, а именнно одиночных и запутанных фотонов, благодаря фундаментальному ограничению числа носителей на одном квантовом уровне, строго определенной энергии излучения изолированной точки, быстрому времени экситонной рекомбинации, обеспечивающим высокую эффективность излучения, и простоте интеграции в фотонные схемы [1]. Помимо крайне востребованной квантовой связи на телекоммуникационных длинах волн, рассматриваются такие сферы применения однофотонных источников как реализация кубитов квантово-вычислительных устройств, преодоление классического дифракционного предела в устройствах интерференционной литографии и уменьшение повреждения клеток при микроскопии биологических систем [2-4]. Демонстрация большинства потенциальных применений была осуществлена с использованием гетероструктур с эпитаксиальными КТ InAs/GaAs [5], на основе которых были созданы наиболее яркие источники одиночных фотонов, резонансно накачиваемые через верхние возбужденные уровни [6]. Однако целый ряд применений, включая атмосферные оптические линии связи и высокочувствительную интерферометрическую диагностику, не согласуется с оптимальным для этих точек спектральным диапазоном $(900-1000 \mathrm{~nm})$. Более того, повышение быстродействия систем квантовой оптики требует, как правило, уменьшения рабочих длин волн. Поэтому активно исследуются КТ, излучающие в видимой области спектра, выполненные на основе других материальных систем, в частности соединений групп II-VI [7-10].

С конца 1990-ых годов в фокусе внимания исследователей были II-VI KT, получаемые методом молекулярнопучковой эпитаксии (МПЭ) путем вставки дробного количества монослоев $\mathrm{CdSe}$ или CdTe в матрицу на основе $\mathrm{ZnSe}[11,12]$. На начальном этапе свойства таких КТ во многом напоминали свойства разупорядоченных квантовых ям [7-9]. Однако совершенствование в течении более десяти лет технологии МПЭ позволило создать массивы изолированных II-VI точек с низкой поверхностной плотностью. На основе таких одиночных КТ в нескольких научных группах были созданы источники одиночных фотонов [13]. Однако ожидаемый для широкозонных КТ прогресс на поприще создания однофотонных источников, работающих при комнатной температуре, был весьма скромным. Достигнутые результаты были плохо воспроизводимыми, интенсивность однофотонного излучения была низкой, а корреляционная функция второго порядка была на уровне $g^{(2)}(0) \simeq 0.16$ [8]. Эти результаты не были неожиданными. Интенсивность узких линий экситонной фотолюминесценции (ФЛ) в II-VI KT резко падает с ростом температуры [14] при достаточной, на первый взгляд, энергии локализации носителей. Это объяснялось сильным экситон-фононным 
взаимодействием в широкозонных II-VI точках $[15,16]$. Однако факт получения, при тщательном контроле ростовых параметров, однофотонного излучения из таких КТ при комнатной температуре свидетельствует о том, что должен быть, по крайней мере, еще один фактор, причем не столь общего характера, как экситонфононное взаимодействие.

Мы полагаем, что причина резкого различия между KT III-V и II-VI лежит отчасти в их морфологии. Канонические InAs точки имеют форму, близкую к пирамидальной с четко очерченными границами раздела с окружающей матрицей [17]. Композиционно материал точек однороден, так же как и материал смачивающего слоя, на котором они располагаются. Отличительная черта KT CdSe/ZnSe KT - это одноосная анизотропия: характерные размеры точек $-2-4 \mathrm{~nm}$ в направлении роста и $6-10 \mathrm{~nm}$ в плоскости. Ей сопутствует композиционная неоднородность: содержание $\mathrm{Cd}$ максимально в центре квантовой точки, где оно достигает $80-100 \%$, будучи в $2-3$ раза меньше на краях. Причем, резкость границ различна в направлении роста (не более $2-3$ постоянных решетки) и в плоскости роста, где градиент изменения состава более плавный и только в редких случаях выходит на чистый $\mathrm{ZnSe}$ [18-20]. Форма точек напоминает сплюснутый сфероид, и моделирующий потенциал не может быть выбран жестким. Характерное изображение KT CdSe/ZnSe, полученное просвечивающей электронной микроскопией с высоким разрешением (HR TEM), и ее аппроксимация сплюснутым сфероидом приведены на рис. 1. Усовершенствованные методы эпитаксии, к примеру, метод формирования КТ с температурной активацией [21], уменьшают, но не ликвидируют полностью анизотропию и композиционную неоднородность точек.

Ранее нами было показано, что поверхностная плотность точек определяет ширину полосы ФЛ, поскольку уменьшение расстояния между точками способствует резонансному переносу энергии и селекции излучающих КТ $[21,22]$. Анализ результатов оптических исследований опирался на численный расчет уровней энергии электронов и дырок в сфероидных QDs с гауссовым потенциальным профилем [22]. Теоретическое моделирование плавного потенциала сфероидальных КТ с помощью функции Гаусса и анализ зависимости энергии основного уровня квантования электронов и дырок от параметров потенциала и материала были впервые выполнены в работе [24]. При этом была учтена аксиальная анизотропия КТ, обусловленная разницей ее размеров в плоскости и вдоль оси роста, однако потенциальный барьер для носителей предполагался одинаковым во всех трех направлениях. В рамках такого подхода были исследованы два первых уровня размерного квантования в различных потенциалах [25]. Рассмотрение влияния анизотропии КТ, связанной с композиционной неоднородностью, а именно, с разницей потенциальных барьеров для носителей в плоскости КТ и вдоль оси роста, на локализацию носителей ранее не проводилось.

В данной работе ставится задача рассмотрения локализации электронов и дырок в градиентных сфероидаль-
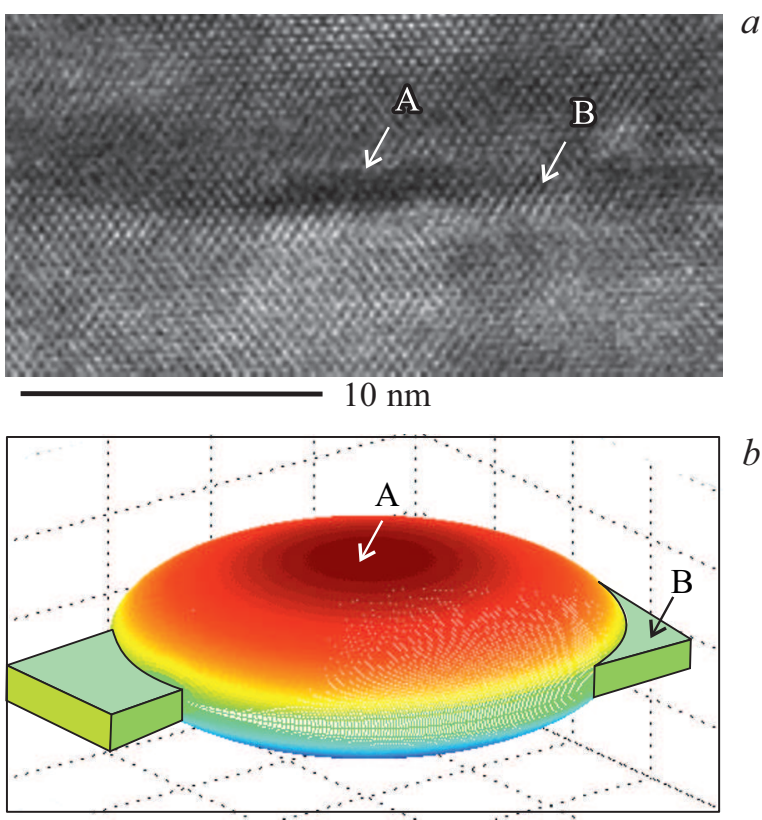

Рис. 1. (a) Типовое изображение квантовой точки $\mathrm{CdSe} / \mathrm{ZnSe}$, выращенной МПЭ, полученное методом просвечивающей электронной микроскопии с высоким разрешением. (b) Схематическое изображение точки в виде сплюснутого сфероида. Области А и В соответствуют высокой (в точке) и низкой (в прилегающих областях) концентрации Сd. (Авторы благодарны С. Рувимову за возможность предоставить НР TEM изображение $\mathrm{CdSe} / \mathrm{ZnSe}$ KT.

ных квантовых точках с учетом различия размеров и высоты барьеров вдоль оси роста структуры и в ее плоскости. В общем случае это соответствует анизотропии КТ трех типов: анизотропии размера КТ, анизотропии потенциального барьера КТ и комбинированной анизотропии. Для трех типов анизотропных КТ будет определены распределение плотности заряда в плоскости и условия локализации носителей заряда внутри КТ. Поскольку наиболее эффективным методом накачки является резонансное возбуждение, будет уделено внимание условиям существования верхних возбужденных уровней в таких объектах.

\section{2. Теоретическая модель}

\section{А. Моделирование потенциального профиля Квантовой точки}

Мы рассматриваем квантовые точки $\mathrm{Cd}_{x} \mathrm{Zn}_{1-x} \mathrm{Se}$ в матрице $\mathrm{ZnSe}$, характеризуемые плавным изменением концентрации $\mathrm{Cd}$ от максимального значения, $x$, в центре точки и до $x^{*}$ за пределами точки. В сферически симметричном случае предполагается что $x^{*}=0$ во всех направлениях, и потенциальный профиль квантовых точек (КТ) для электронов и дырок моделируется с помощью 
сферически симметричного потенциала Гаусса как

$$
V_{\mathrm{e}, \mathrm{h}}(r)=V_{\mathrm{off}}^{\mathrm{e}, \mathrm{h}}\left(x-x^{*}\right)\left[1-\exp \left(-\frac{4 r^{2}}{d^{2}}\right)\right],
$$

Здесь $V_{\mathrm{off}}^{\mathrm{e}, \mathrm{h}}\left(x-x^{*}\right)$ - потенциальные барьеры для электронов и дырок, зависящие от концентрации $\mathrm{Cd}$ в центре точки $x$, a $d-$ характерный размер КТ. Рассчитанные согласно [26] зависимости $V_{\text {off }}^{\mathrm{e}, \mathrm{h}}(x)=\eta_{\mathrm{e}, \mathrm{h}}\left[E_{\mathrm{g}}(0)-E_{\mathrm{g}}(x)\right]$ при $\eta_{\mathrm{e}}=0.75, \eta_{\mathrm{h}}=0.25$ и $E_{\mathrm{g}}(x)=(1-x) E_{\mathrm{g}}(0)+x E_{\mathrm{g}}(1)-0.387 x(1-x)$ показаны на рисунке рис. 2. Здесь $E_{\mathrm{g}}(0)=2.82 \mathrm{eV}$ - величина запрещенной зоны $\mathrm{ZnSe}$ и $E_{\mathrm{g}}(1)=1.74 \mathrm{eV}$ - величина запрещенной зоны $\mathrm{CdSe}$ при температуре $0 \mathrm{~K}$. Если концентрация $\mathrm{Cd}$ снаружи точки равна $x^{*}$, потенциальный барьер может быть найден как $V_{0}^{\mathrm{e}, \mathrm{h}}=$ $=V_{\mathrm{off}}^{\mathrm{e}, \mathrm{h}}\left(x-x^{*}\right)=\eta_{\mathrm{e}, \mathrm{h}}\left[E_{\mathrm{g}}\left(x^{*}\right)-E_{\mathrm{g}}(x)\right]=V_{\mathrm{off}}^{\mathrm{e}, \mathrm{h}}(x)-V_{\mathrm{off}}^{\mathrm{e}, \mathrm{h}}\left(x^{*}\right)$. Таким образом, варьирование $x^{*} \neq 0$ позволяет учесть ненулевую концентрацию $\mathrm{Cd}$ между КТ в плоскости структуры, приводящую к понижению потенциального барьера по сравнению с потенциальным барьером вдоль оси роста.

Потенциал (1) соответствует нулевой энергии в центре КТ и энергии $V_{\text {off }}^{\mathrm{e}, \mathrm{h}}$ для электронов и дырок, соответственно, на расстоянии примерно $d=2 a$ от центра точки. Мы будем считать, что $a-$ эффективный радиус точки (соответствует потенциальной энергии примерно $\left.0.6 V_{\text {off }}^{\mathrm{e}, \mathrm{h}}\right), d$ имеет смысл характерного диаметра квантовой точки. На расстоянии $r \ll a$ от центра КТ потенциал (1) может быть аппроксимирован потенциалом гармонического осциллятора:

$$
V_{\mathrm{e}, \mathrm{h}}(r) \approx \frac{\kappa_{\mathrm{e}, \mathrm{h}} r^{2}}{2},
$$

где $\kappa_{\mathrm{e}, \mathrm{h}}=2 V_{\mathrm{off}}^{\mathrm{e}, \mathrm{h}} / a^{2}-$ жесткости соответствующих осцилляторов.

В работе мы используем приближение сильного размерного квантования, в котором электрон и дырка кван-

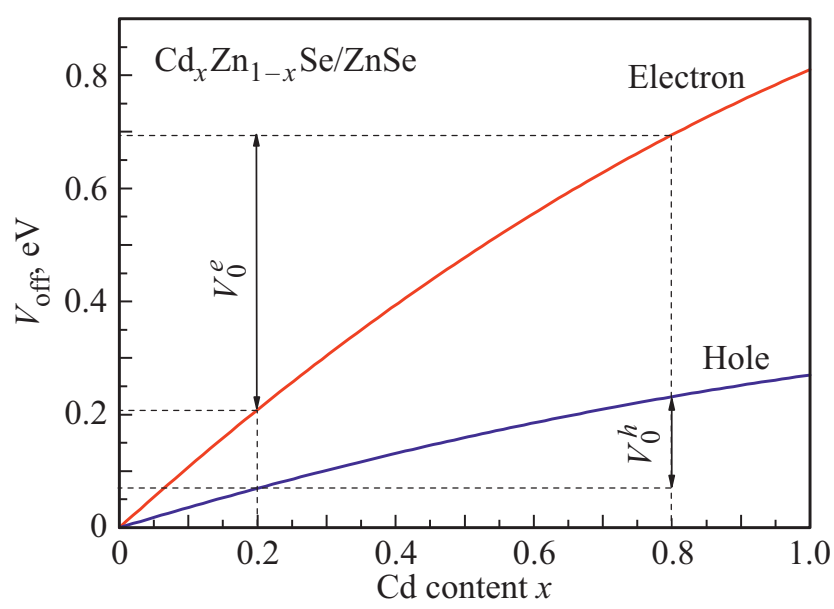

Рис. 2. Зависимость $V_{\text {off }}^{\mathrm{e}, \mathrm{h}}$ для электронов и дырок от концентрации кадмия $x$ в центре квантовой точки $\mathrm{Cd}_{x} \mathrm{Zn}_{1-x} \mathrm{Se} / \mathrm{ZnSe}$. Стрелками показано определение потенциального барьера $V_{0}^{\mathrm{e}, \mathrm{h}}$ для электронов и дырок в плоскости при значениях концентрации $\mathrm{Cd} x=0.8$ в центре КТ и $x^{*}=0.2$ между КТ в плоскости. туются в квантовой точке независимо, а кулоновское взаимодействие можно рассмотривать как возмущение. Мы моделируем сплюснутые анизотропные КТ трех характерных типов, следующими способами:

1) Предполагаем что концентрация $\mathrm{Cd}$ снаружи КТ $x^{*}=0$ во всех направлениях, однако характерный размер КТ в плоскости больше, чем характерный размер вдоль направления $z$. В этом случае потенциалы могут быть записаны как

$$
V_{\mathrm{e}, \mathrm{h}}(r)=V_{\mathrm{off}}^{\mathrm{e}, \mathrm{h}}(x)\left[1-\exp \left(-\frac{x^{2}+y^{2}}{a_{x}^{2}}-\frac{z^{2}}{a_{z}^{2}}\right)\right],
$$

В центре КТ такой потенциал имеет вид

$$
V_{\mathrm{e}, \mathrm{h}}^{d}(r) \approx \kappa_{\mathrm{e}, \mathrm{h}}^{x} \frac{x^{2}+y^{2}}{2}+\kappa_{\mathrm{e}, \mathrm{h}}^{z} \frac{z^{2}}{2},
$$

где $\kappa_{\mathrm{e}, \mathrm{h}}^{x}=2 V_{\mathrm{off}}^{\mathrm{e}, \mathrm{h}} / a_{x}^{2}$ и $\kappa_{\mathrm{e}, \mathrm{h}}^{z}=2 V_{\mathrm{off}}^{\mathrm{e}, \mathrm{h}} / a_{z}^{2}$.

2) Предполагаем, что характерный размер КТ одинаковый во всех направлениях, однако распределение концентрации анизотропно: концентрация $\mathrm{Cd}$ снаружи КТ $x^{*}=0$ в направлении $z$ и $x^{*} \neq 0$ в плоскости. Этот случай мы моделируем следующим потенциалом:

$$
\begin{gathered}
V_{\mathrm{e}, \mathrm{h}}(r)=V_{0}^{\mathrm{e}, \mathrm{h}}\left[1-\exp \left(-\frac{r^{2}}{a^{2}}\right)\right]+\Delta V^{\mathrm{e}, \mathrm{h}}\left[1-\exp \left(-\frac{z^{2}}{a^{2}}\right)\right] \\
V_{0}^{\mathrm{e}, \mathrm{h}}+\Delta V^{\mathrm{e}, \mathrm{h}}=V_{\text {off }}^{\mathrm{e}, \mathrm{h}}
\end{gathered}
$$

Здесь $\Delta V^{\mathrm{e}, \mathrm{h}}=V_{\mathrm{off}}^{\mathrm{e}, \mathrm{h}}\left(x^{*}\right) \quad$ и $\quad V_{0}^{\mathrm{e}, \mathrm{h}}=\eta_{\mathrm{e}, \mathrm{h}}\left[E_{\mathrm{g}}\left(x^{*}\right)-E_{\mathrm{g}}(x)\right]=$ $=V_{\mathrm{off}}^{\mathrm{e}, \mathrm{h}}(x)-V_{\mathrm{off}}^{\mathrm{e}, \mathrm{h}}\left(x^{*}\right)-$ потенциальный барьер в плоскости. В центре КТ такой потенциал также имеет вид (4), где $\kappa_{\mathrm{e}, \mathrm{h}}^{x}=2 V_{0}^{\mathrm{e}, \mathrm{h}} / a^{2}$ и $\kappa_{\mathrm{e}, \mathrm{h}}^{z}=2 V_{\mathrm{off}}^{\mathrm{e}, \mathrm{h}} / a^{2}$.

3) В третьем случае мы комбинируем анизотропию размера, состава и выбираем потенциальный профиль в виде:

$$
\begin{aligned}
V_{\mathrm{e}, \mathrm{h}}(r)= & V_{0}^{\mathrm{e}, \mathrm{h}}\left[1-\exp \left(-\frac{x^{2}+y^{2}}{a_{x}^{2}}-\frac{z^{2}}{a_{z}^{2}}\right)\right] \\
+ & \Delta V^{\mathrm{e}, \mathrm{h}}\left[1-\exp \left(-\frac{z^{2}}{a_{z}^{2}}\right)\right], \\
& V_{0}^{\mathrm{e}, \mathrm{h}}+\Delta V^{\mathrm{e}, \mathrm{h}}=V_{\mathrm{off}}^{\mathrm{e}, \mathrm{h}}
\end{aligned}
$$

В центре КТ такой потенциал также имеет вид (4), где $\kappa_{\mathrm{e}, \mathrm{h}}^{x}=2 V_{0}^{\mathrm{e}, \mathrm{h}} / a_{x}^{2}$ и $\kappa_{\mathrm{e}, \mathrm{h}}^{z}=2 V_{\mathrm{off}}^{\mathrm{e}, \mathrm{h}} / a_{z}^{2}$.

При решении задачи мы также считаем, что массы носителей заряда не зависят от координаты во всем объеме КТ, что оправдано в силу малой разницы между эффективными массами электрона и дырки в кубических модификациях CdSe и ZnSe. Параметры структур $\mathrm{Cd}_{x} \mathrm{Zn}_{1-x} \mathrm{Se} / \mathrm{ZnSe}$ для некоторых значений $x$, представлены в таблице.

\section{В. Расчет уровней энергии электронов и дырок}

Уровни энергии электронов, $E_{\mathrm{e}}$, и дырок, $E_{\mathrm{h}}$, в КТ с соответствующими потенциальными профилями $V_{\mathrm{e}, \mathrm{h}}^{\mathrm{sp}, \mathrm{ax}}(r)$ 
Параметры структур $\mathrm{Cd}_{x} \mathrm{Zn}_{1-x} \mathrm{Se} / \mathrm{ZnSe}$ при температуре $0 \mathrm{~K}$. Значения эффективных масс получено при помощи линейной интерполяции между значениями для чистых $\mathrm{ZnSe}$ и CdSe.

\begin{tabular}{c|c|c|c|c|c|c|c}
\hline$x$ & $E_{\mathrm{g}}(x), \mathrm{eV}$ & $V_{\text {off }}^{\mathrm{e}}, \mathrm{eV}$ & $V_{\text {off }}^{\mathrm{h}}, \mathrm{eV}$ & $m_{\mathrm{e}} / m_{0}$ & $m_{\mathrm{l}} / m_{0}$ & $m_{\mathrm{h}} / m_{0}$ & $\beta$ \\
\hline 0. & 2.82 & 0.0 & 0.0 & 0.16 & 0.145 & 0.6 & 0.24 \\
\hline 0.2 & 2.46 & 0.21 & 0.07 & 0.154 & 0.145 & 0.57 & 0.254 \\
\hline 0.5 & 2.18 & 0.48 & 0.16 & 0.145 & 0.145 & 0.525 & 0.275 \\
\hline 0.8 & 1.9 & 0.69 & 0.23 & 0.136 & 0.145 & 0.48 & 0.3 \\
\hline 1 & 1.74 & 0.81 & 0.27 & 0.13 & 0.145 & 0.45 & 0.32
\end{tabular}

могут быть найдены в результате решения уравнений Шредингера

$$
\left[\hat{H}_{\mathrm{e}, \mathrm{h}}+V_{\mathrm{e}, \mathrm{h}}(r)\right] \Psi_{\mathrm{e}, \mathrm{h}}(\mathbf{r})=E_{\mathrm{e}, \mathrm{h}} \Psi_{\mathrm{e}, \mathrm{h}}(\mathbf{r}) .
$$

Гамильтониан электрона $\hat{H}_{\text {e }}$ рассмотрен в рамках однозонной модели с изотропной эффективной массой:

$$
\hat{H}_{\mathrm{e}}=\frac{\hbar^{2} \hat{k}^{2}}{2 m_{\mathrm{e}}},
$$

где $\hat{k}=-i \nabla-$ оператор волнового вектора, и $m_{\mathrm{e}}-$ эффективная масса электрона. В сферически симметричном случае и при больших значениях $V_{\text {off }}^{\text {e }}$ собственные состояния такого гамильтониана приближаются к собственным состояниям гармонического осциллятора [24]. Для того, чтобы иметь единые единицы измерения длины и энергии, выбираем в качестве единиц измерения длину $L_{\mathrm{e}}$ и энергию $E_{0}^{\mathrm{e}}$ осциллятора с жесткостью $\kappa=\kappa_{z}$ в центре КТ:

$$
\begin{gathered}
L_{\mathrm{e}}^{2}=\frac{\hbar}{\sqrt{k_{e} m_{e}}}=a^{2} \sqrt{\frac{E_{k}^{\mathrm{e}}}{V_{\mathrm{off}}^{\mathrm{e}}}}, \quad E_{k}^{\mathrm{e}}=\frac{\hbar^{2}}{2 m_{e} a^{2}}, \\
E_{0}^{\mathrm{e}}=\frac{3}{2} \frac{\hbar^{2}}{m_{e} L_{\mathrm{e}}^{2}}=3 V_{\mathrm{off}}^{\mathrm{e}} \sqrt{\frac{E_{k}^{\mathrm{e}}}{V_{\mathrm{off}}^{\mathrm{e}}}} .
\end{gathered}
$$

Отметим, что зависимость энергии уровней электрона от размера квантовой точки $a$ содержится в зависимости характерной энергии осциллятора $E_{0} \propto 1 / a$.

Состояния дырки в вершине валентной зоны $\Gamma_{8}$ кубической модификации полупроводника II-VI могут быть описаны в рамках четырехзонного Гамильтониана Латтинжера $[27,28]$ :

$$
\hat{H}_{\mathrm{h}}=\frac{\hbar^{2}}{2 m_{0}}\left[\left(\gamma_{1}+\frac{5}{2} \gamma\right) \hat{k}^{2}-2 \gamma(\hat{\mathbf{k}} \mathbf{J})^{2}\right] .
$$

Здесь $\mathbf{J}$ - оператор собственного углового момента дырки $J=3 / 2, \gamma_{1}$ и $\gamma=\left(2 \gamma_{2}+3 \gamma_{3}\right) / 5$ - параметры Латтинжера, связанные с массами легкой, $m_{1}$, и тяжелой, $m_{\mathrm{h}}$, дырок соотношениями $m_{\mathrm{l}, \mathrm{h}}=m_{0} /\left(\gamma_{1} \pm 2 \gamma\right)\left(m_{0}-\right.$ масса свободного электрона). Здесь мы пренебрегаем гофрировкой валентной зоны и используем сферическое приближение для гамильтониана Латтинжера, так как малые эффекты кубической симметрии кристалла не имеют существенного влияния на положение уровней дырки.

В случае сферически симметричного потенциала квантовой точки основное состояние дырки характеризуется полным угловым моментом $\mathbf{j}=3 / 2$ и четырехкратно вырождено по значению проекции момента $M=3 / 2,1 / 2,-1 / 2,-3 / 2$ на ось $z$ [28]. Энергия вырожденного состояния дырки в КТ, как в случае плавного, так и в случае жесткого потенциалов, может быть найдена в характерных единицах энергии размерного квантования в виде универсальной зависимости от отношения масс легкой и тяжелой дырок $\beta=m_{\mathrm{l}} / m_{\mathrm{h}}[24,29,30]$. В случае $\beta=1$, что соответствует простой зоне, задача нахождения уровней дырки полностью аналогична задаче об уровнях электрона, если использовать в качестве единиц измерения единицы, аналогичные электронным с заменой $m_{\mathrm{e}}$ на массу тяжелой дырки $m_{\mathrm{h}}$ :

$$
L_{\mathrm{h}}^{2}=\frac{\hbar}{\sqrt{k_{h} m_{h}}}, \quad E_{0}^{\mathrm{h}}=\frac{3}{2} \frac{\hbar^{2}}{m_{h} L_{\mathrm{h}}^{2}} .
$$

В безразмерных единицах рассматриваемые потенциалы, $v=V_{e, h} / E_{0}$, могут быть записаны в общем виде как

$$
\begin{aligned}
v\left(r_{\mathrm{e}, \mathrm{h}}\right)= & \frac{1}{\xi_{p}} v_{\mathrm{off}}\left[1-\exp \left(-\frac{\rho_{\mathrm{e}, \mathrm{h}}^{2}}{3 \xi_{s} v_{\mathrm{off}}}-\frac{z_{\mathrm{e}, \mathrm{h}}^{2}}{3 v_{\mathrm{off}}}\right)\right] \\
& +\frac{\xi_{p}-1}{\xi_{p}} v_{\mathrm{off}}\left[1-\exp \left(-\frac{z_{\mathrm{e}, \mathrm{h}}^{2}}{3 v_{\mathrm{off}}}\right)\right] .
\end{aligned}
$$

Здесь $\rho_{\mathrm{e}, \mathrm{h}}^{2}=\left(x^{2}+y^{2}\right) / L_{\mathrm{e}, \mathrm{h}}^{2}, \quad z_{\mathrm{e}, \mathrm{h}}=z / L_{\mathrm{e}, \mathrm{h}}, \quad v_{\mathrm{off}}=V_{\mathrm{off}}^{\mathrm{e}, \mathrm{h}} / E_{0}^{\mathrm{e}, \mathrm{h}}-$ безразмерная высота потенциального барьера, и параметр анизотропии $\xi_{p} \xi_{s}=\xi^{\mathrm{e}, \mathrm{h}}=\kappa_{z}^{\mathrm{e}, \mathrm{h}} / \kappa_{x}^{\mathrm{e}, \mathrm{h}}>1$ для сплюснутых КТ.

Безразмерные потенциальные профили, моделируемые с помощью выражения (13) при $v_{\text {off }}=2$, показаны на рис. 2,a. Для наглядности, мы выбрали КТ с одинаковыми потенциалами вдоль оси роста структуры $z$. Параметры анизотропных точек в плоскости структуры выбраны так, чтобы анизотропия жесткости потенциалов КТ вблизи их центра была одинаковой: $\kappa_{\mathrm{e}, \mathrm{h}}^{x}=\kappa_{\mathrm{e}, \mathrm{h}}^{y}=\kappa_{\mathrm{e}, \mathrm{h}}^{z} / \xi$ для $\xi=\xi_{p} \xi_{s}=2$. Черная сплошная кривая показывает сферически-симметричный потенциал. Красными (точки), синими (штрихи) и розовыми (штрих-пунктир) кривыми показаны зависимости потенциалов от безразмерной координаты вдоль оси $x$ для $\xi_{s}=2$ (анизотропия только размера КТ), $\xi_{s}=\xi_{p}=\sqrt{2}$ (комбинированная анизотропия), и $\xi_{p}=2$ (анизотропия только потенциального барьера КТ).

Для рассматриваемых потенциалов $v(r)$, как сферического так и аксиально симметричного, точного аналитического решения задачи ни для $\beta=1$, ни для $\beta<1$ не существует. Мы применили численный метод решения, описанный подробно в работе [24] и применимый как для 
сферически, так и для аксиально симметричного потенциалов. Для нахождения собственных энергий и функций гамильтониана (7) мы диагонализуем его матрицу, вычисленную на ортонормированном базисе волновых функций аксиально анизотропного гармонического осциллятора. Таким образом, при достаточном размере базиса можно получить с высокой точностью не только энергию и волновую функцию основного состояния но и энергии и волновые функции возбужденных состояний.

\section{3. Результаты}

\section{А. Условия локализации в сферических КT}

Для модельного сферически-симметричного потенциала с потенциальным барьером $v_{\text {off }}=2$ на рис. 3, $a$

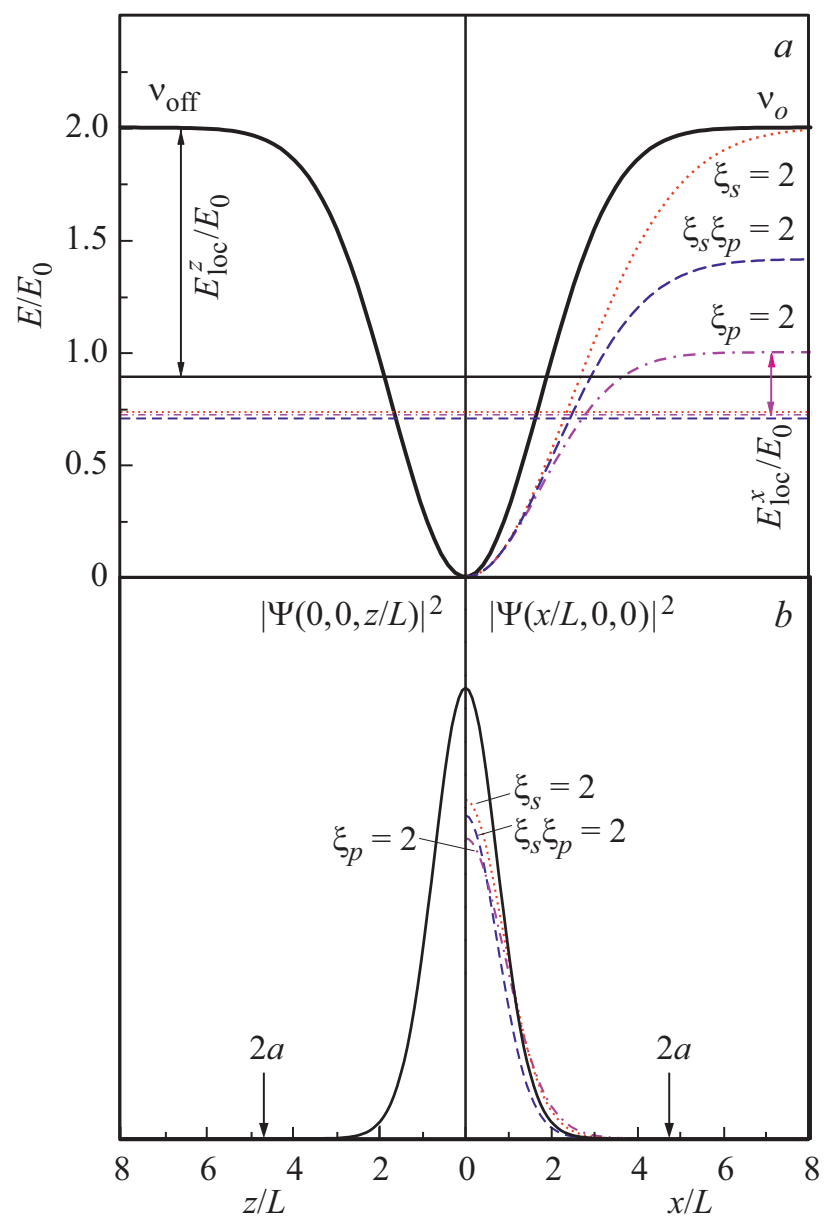

Pис. 3. (a) Безразмерные потенциальные профили аксиально анизотропных КТ, моделируемые с помощью выражения (13) при $v_{\text {off }}=2$, и уровни энергии в единицах $E_{0}$, рассчитанные для $\beta=1$. Горизонтальные линии показывают соответствующие энергии. Левая панель - профиль потенциала КТ вдоль оси $z$, правая панель - профиль потенциала КТ вдоль оси $x$ (в плоскости структуры); (b) Пространственное распределение квадрата модуля волновой функции для потенциалов, показанных на панеле $(a)$. Стрелками показан удвоенный характерный размер КТ вдоль оси $z$.

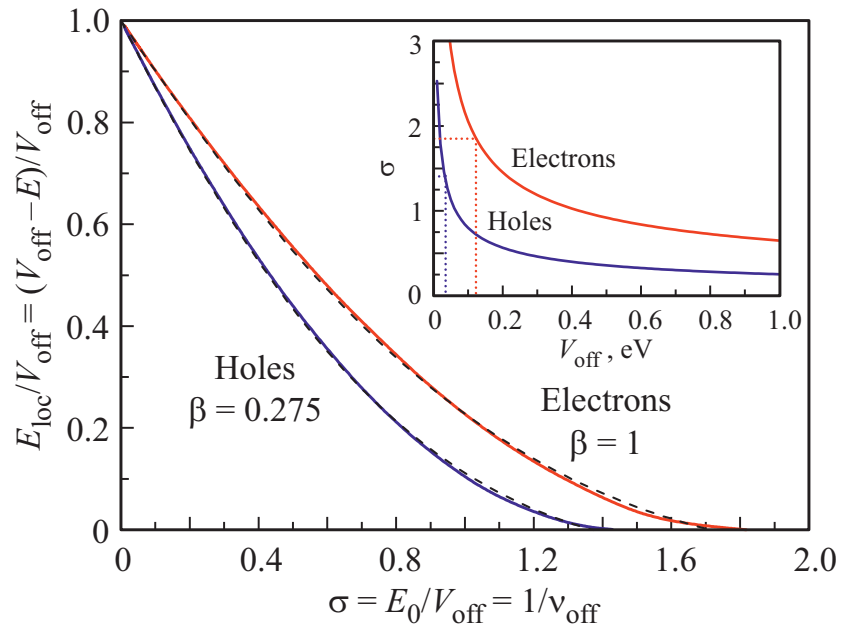

Рис. 4. Засисимости $E_{\mathrm{e}, \mathrm{h}}^{\mathrm{loc}} / V_{\mathrm{off}}^{\mathrm{e}, \mathrm{h}}=\left(V_{\mathrm{off}}^{\mathrm{e}, \mathrm{h}}-E_{\mathrm{e}, \mathrm{h}}\right) / V_{\mathrm{off}}^{\mathrm{e}, \mathrm{h}}$ для электронов и дырок $(\beta=0.275)$ от безразмерного параметра $\sigma^{\mathrm{e}, \mathrm{h}}=E_{0}^{\mathrm{e}, \mathrm{h}} / V_{\mathrm{off}}^{\mathrm{e}, \mathrm{h}}=3 \sqrt{E_{k}^{\mathrm{e}, \mathrm{h}} / V_{\text {off }}^{\mathrm{e}, \mathrm{h}}} \cdot$ Штриховыми линиями показаны аппроксимирующие зависимости согласно $\quad E_{\mathrm{loc}}^{\mathrm{e}} / V_{\mathrm{off}}^{\mathrm{e}} \approx 1-\sigma /\left(1+0.125 \sigma+0.17 \sigma^{2}\right) \quad$ и $\quad E_{\mathrm{loc}}^{\mathrm{h}} / V_{\mathrm{off}}^{\mathrm{h}} \approx 1$ $-1.335 \sigma /\left(1+0.22 \sigma+0.27 \sigma^{2}\right)$. На вставке показаны зависимости безразмерного параметра $\sigma=1 / v_{\text {off }}$ для электронов с массой $m_{e}=0.5 m_{0}$ (красная кривая) и дырок с массой $m_{h}=0.53 m_{0} \quad$ (синяя кривая) от величины потенциального барьера $V_{\text {off }}$ в диапазоне от 0.1 до $1 \mathrm{eV}$ для КТ диаметром $d=3 \mathrm{~nm}$. Пунктирные линии на вставке показывают пороговые значения $V_{\text {off }}$ и $\sigma$ при которых локализация электрона и дырки пропадают.

сплошной кривой показаны безразмерный уровень энергии основного состояния электрона $\epsilon=E_{\mathrm{e}} / E_{0}$ и его безразмерная энергия локализации $E_{\mathrm{loc}} / E_{0}=v_{\text {off }}-E / E_{0}$. На рис. $3, b$ показано распределение плотности вероятности для электрона в такой КТ. Из рисунка можно видеть, что волновая функция хорошо локализована на размере удвоенного характерного размера квантовой точки.

Зависимости энергии основного уровня электронов и дырок $\epsilon=E^{\mathrm{e}, \mathrm{h}} / E_{0}^{\mathrm{e}, \mathrm{h}}$ от величины потенциального барьера $v_{\text {off }}=V_{\text {off }}^{\mathrm{e}, \mathrm{h}} / E_{0}^{\mathrm{e}, \mathrm{h}}$ в сферически симметричном потенциале Гаусса были представлены в работе [24]. Здесь мы представляем обобщение анализа условий локализации электронов и дырок в сферических КТ $\mathrm{Cd}_{x} \mathrm{Zn}_{1-x} \mathrm{Se} / \mathrm{ZnSe}$ разного диаметра $d$ и при разных значениях $x$ в центре КТ, соответсвующих разным значениям $V_{\text {off }}^{\mathrm{e} \text {. }}$. На рис. 4 построены безразмерные зависимости $E_{\mathrm{loc}}^{\mathrm{e}, \mathrm{h}} / V_{\mathrm{off}}^{\mathrm{e}, \mathrm{h}}=\left(V_{\mathrm{off}}^{\mathrm{e}, \mathrm{h}}-E^{\mathrm{e}, \mathrm{h}}\right) / V_{\mathrm{off}}^{\mathrm{e}, \mathrm{h}}$ для электронов и дырок (с $\beta=0.275$, что соответствует $x=0.5)$ от безразмерного параметра $\sigma=1 / v_{\text {off }}=E_{0}^{\mathrm{e}, \mathrm{h}} / V_{\text {off }}^{\mathrm{e}, \mathrm{h}}=3 \sqrt{E_{\mathrm{k}}^{\mathrm{e}, \mathrm{h}} / V_{\text {off }}^{\mathrm{e}, \mathrm{h}}}$. Результаты точных расчетов хорошо аппроксимируются зависимостями $E_{\mathrm{loc}}^{\mathrm{e}} / V_{\mathrm{off}}^{\mathrm{e}} \approx 1-\sigma /\left(1+0.125 \sigma+0.17 \sigma^{2}\right)$ для электронов и $E_{\mathrm{loc}}^{\mathrm{h}} / V_{\mathrm{off}}^{\mathrm{h}} \approx 1-1.335 \sigma /\left(1+0.22 \sigma+0.27 \sigma^{2}\right)$ для дырок (при $\beta=0.275$ ).

Видно, что электроны и дырки хорошо локализуются в КТ при выполнении условия $\sigma<1 \quad\left(E_{0}<V\right.$ 
или $\left.v_{\text {off }}>1\right)$. Для конкретных КТ энергии локализации электронов и дырок можно оценить, определив значение параметров $\sigma^{\mathrm{e}, \mathrm{h}}=3 \sqrt{E_{\mathrm{k}}^{\mathrm{e}, \mathrm{h}} / V_{\text {off }}^{\mathrm{e}, \mathrm{h}}}$ для заданных размерных значений $V_{\text {off }}$ и диаметра $d$. На вставке на рис. 4 построены зависимости $\sigma^{\mathrm{e}, \mathrm{h}}$ от $V_{\text {off }}$ в КТ диаметром $d=3 \mathrm{~nm}$ для электронов с $m_{\mathrm{e}} / m_{0}=0.15$ и дырок с $m_{\mathrm{h}} / m_{0}=0.53$, соотвественно. Для КТ с другим характерным диаметром параметр $\sigma \propto d$, и значение $E_{0}$ может быть найдено как $\sigma V_{\text {off. }}$

Локализованное состояние электронов или дырок в КТ исчезает при $\sigma_{e}>1.8$ и $\sigma_{h}>1.4$ соответственно. Это соответствует пороговым значениям потенциальных барьеров $v_{\mathrm{off}}^{\mathrm{e}} \approx 0.56$ и $v_{\mathrm{off}}^{\mathrm{h}} \approx 0.71$ или $V_{\mathrm{off}}^{\mathrm{e}} \approx 0.13 \mathrm{eV}$ и $V_{\mathrm{off}}^{\mathrm{h}} \approx 0.035 \mathrm{eV}$ в КТ с характерным диаметром $d=3 \mathrm{~nm}$ (показано пунктирными линиями на вставке на рис. 4). При увеличении $d$ пороговые значения потенциальных барьеров, ниже которых локализация носителей отсутвует, или понижаются, что, очевидно, связано с уменьшением характерной энергии размерного квантования с увеличением размера квантовой точки.

\section{В. Влияние анизотропии размера КT на локализацию носителей}

Наличие аксиальной анизотропии размера КТ, характеризуемой параметром $\xi_{s}=d_{x}^{2} / d_{z}^{2}$ приводит к понижению уровня энергии размерного квантования электронов и дырок по сравнению с уровнем в сферическисимметричной КТ с усредненным диаметром $d_{s}$, определяемом условием $d_{z}^{2} / d_{s}^{2}=\left(\xi_{s}+2\right) /\left(3 \xi_{s}\right)$ [24] в случае $\xi_{s}>1$ (сплюснутые КТ) по сравнению с уровнем в сферически-симметричной КТ с диаметром $d=d_{z}$. На рис. 3 показано положение уровня энергии электрона в аксиально-анизотропном потенциале с $\xi_{s}=2$, и видно его понижение по сравнению со сферическисимметричным потенциалом, при этом энергия локализация электрона возрастает.

Для оценки порога локализации в аксиальноанизотропных КТ с параметром $\xi_{s}$ можно воспользоваться зависимостями, показанными на рис. 4 для сферически-симметричной КТ с диаметром $d_{s}$. Энергии локализации электронов и дырок, определенные для параметров $\sigma_{\mathrm{e}, \mathrm{h}}$, вычисленных с $d=d_{s}$, являются оценкой снизу. Оценку сверху можно получить, определив энергии локализации для параметров $\sigma_{\mathrm{e}, \mathrm{h}}$, вычисленных c $d=d_{x}$.

Дополнительно, понижение симметрии при аксиальной анизотропии приводит к расщеплению четырехкратно вырожденного основного уровня дырки $E_{\mathrm{h}}$ на состояния с проекцией момента на ось роста структуры $M= \pm 3 / 2$ (тяжелые дырки с уровнем энергии $E_{\mathrm{hh}}$ ) и $M= \pm 1 / 2$ (легкие дырки с уровнем энергии $E_{\mathrm{lh}}$ ), причем в сплюснутых КТ основным является состояние тяжелых дырок. При заданной геометрической анизотропии КТ, характеризуемой параметром $\xi_{s}=d_{x}^{2} / d_{z}^{2}$, относительное расщепление $\Delta_{\mathrm{ax}} / E_{\mathrm{h}}^{\text {loc }}=\left(E_{\mathrm{lh}}-E_{\mathrm{hh}}\right) / E_{\mathrm{h}}^{\text {loc }}$ тем больше, чем меньше значение параметра $\sigma_{\mathrm{h}}=1 / v_{\text {off }}$ для дырок.

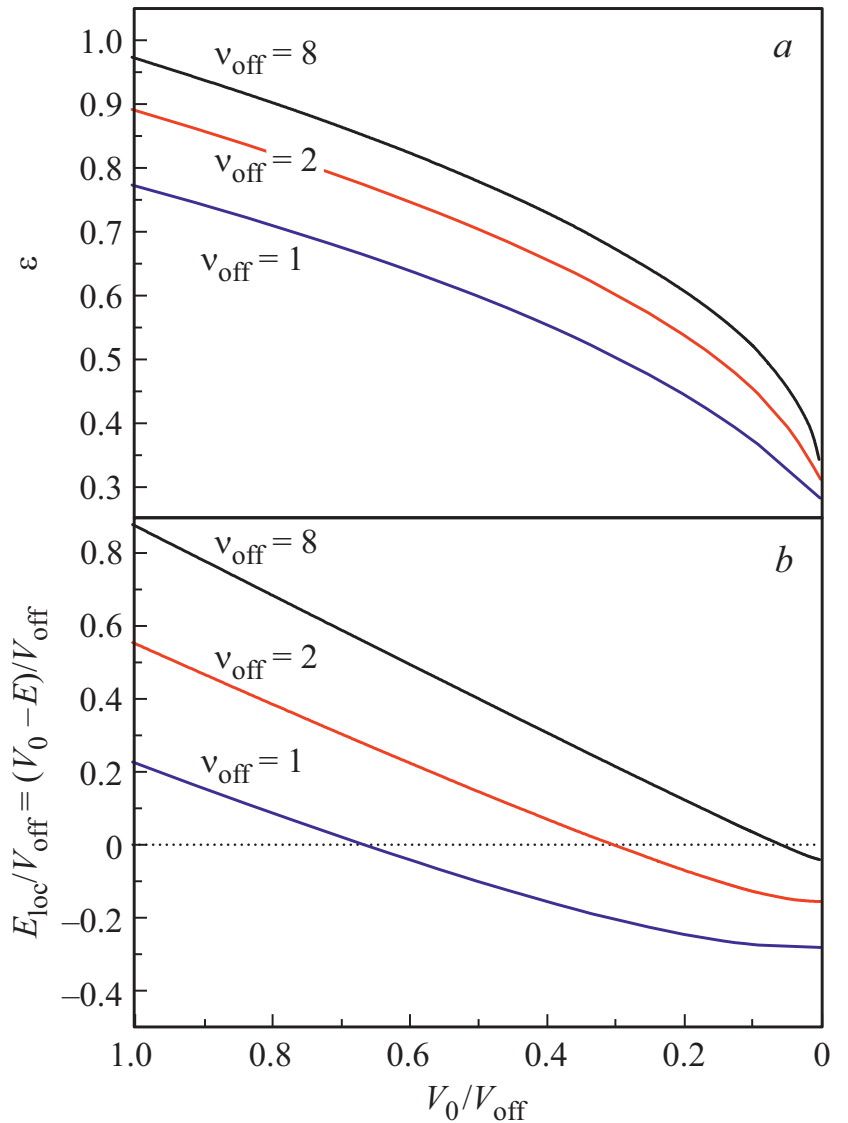

Рис. 5. Зависимость безразмерной энергии уровня электронов $(a)$ и энергии локализации электрона в плоскости КТ $(b)$ от параметра анизотропии потенциального барьера $V_{0} / V_{\text {off }}=1 / \xi_{p}$ для трех значений безразмерного барьера вдоль оси $z \quad v_{\text {off }}=1,2$ и 8 . Горизонтальная пунктирная линия соответствует порогу локализации электронов в плоскости структуры.

\section{С. Влияние анизотропии потенциального барьера КТ на локализацию носителей}

Наличие аксиальной анизотропии потенциального барьера, как и в случае аксиальной анизотропии размера КТ, приводит к понижению уровней размерного квантования электрона и дырки и снятию четырехкратного вырождения уровня дырки. При этом, как видно из рис. 3, a, понижение уровня энергии в потенциалах с заданным значением $\xi=\xi_{s} \xi_{p}$ сопоставимо между собой. Однако в случае $\xi_{p}>1$, барьер в плоскости структуры понижается и понижение уровня энергии сопровождается уменьшением энергии локализации $E_{\mathrm{loc}}=\left(V_{0}-E\right)$ в плоскости КТ. При этом, как видно из рис. $3, b$, вероятность найти электрон за пределами КТ остается ничтожно малой при условии положительной энергии локализации.

На рис. 5, а показана зависимость безразмерного уровня энергии $\varepsilon$ электронов в КТ от анизотропии потенциального барьера $V_{0} / V_{\text {off }}=1 / \xi_{p}$ при сферически симметричном размере КТ $\left(\xi_{s}=1\right)$ и при трех значе- 


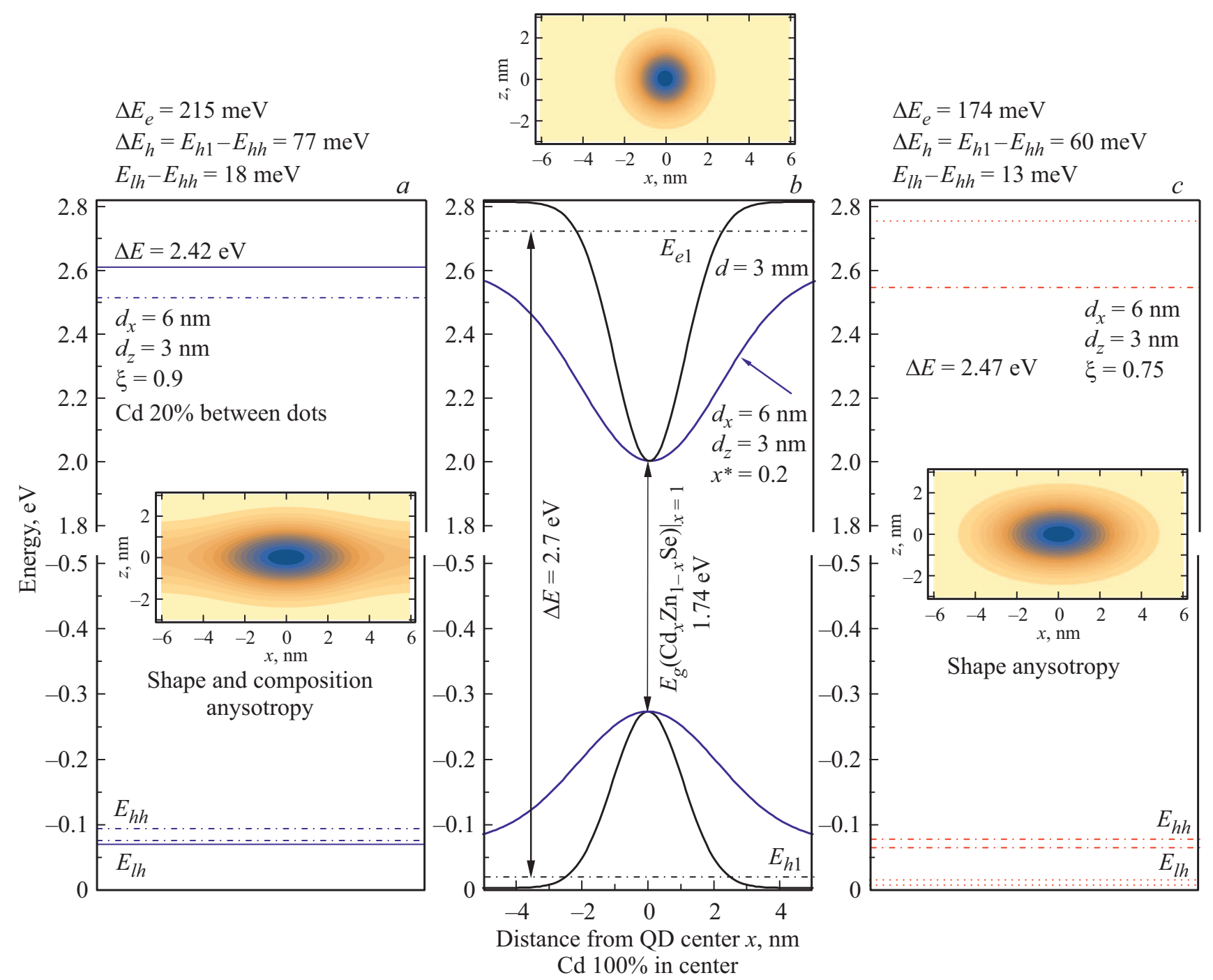

Рис. 6. Потенциальные профили электронов и дырок в сплюснутых квантовых точках $\mathrm{Cd}_{x} \mathrm{Zn}_{1-x} \mathrm{Se} / \mathrm{ZnSe}_{\text {для }}$ значения концентрации $\mathrm{Cd} x=1$ в центре КТ и фиксированного размера КТ в направлении оси $z \quad d_{z}=d=3 \mathrm{~nm}$. Горизонтальные штрихпунктирные линии показывают основные уровни энергии электронов и дырок для локализованных состояний, пунктирные линии на панели $(c)$ - возбужэденные уровни.

ниях безразмерного потенциального барьера $v_{\text {оff }}$ вдоль оси роста структуры. При исчезновении потенциального барьера в плоскости $V_{0}=0$ и $\xi_{p} \gg 1$, уровни энергии стремятся к энергии размерного квантования в двумерной квантовой яме с барьером $V_{\text {off. При этом }}$ энергия локализации носителя вдоль оси $z$, определенная как $E_{\text {loc }}^{z}=\left(V_{\text {off }}-E\right)$ возрастает при фиксированном значении $V_{\text {off }}$, а энергия локализации носителя в плоскости $E_{\mathrm{loc}}=\left(V_{0}-E\right)$ убывает. На рис. $5, b$ показана зависмость энергии локализации носителей в плоскости от анизотропии потенциального барьера $V_{0} / V_{\text {off }}=1 / \xi_{p}$. Видно, что чем больше $v_{\text {off, }}$ тем при меньших значениях $v_{0}$ возможна локализация носителя в плоскости. При $v_{\text {off }}=1$, порог локализации в плоскости соответсвует значению $v_{0} \approx 0.65$ и $\sigma \approx 1.54$, в то время как при $v_{\text {off }}=2$, порог локализации в плоскости соответсвует значению $v_{0} \approx 0.55$ и $\sigma \approx 1.8$, что совпадает с поро- гом локализации, полученным ранее для сферическисимметричных КТ. При отрицательной энергия локализации вероятность обнаружить носитель между квантовыми точками в плоскости возрастает экспоненциально. Однако в области положительных, хотя и малых энергий локализации вероятность обнаружить электоны и дырки на расстоянии большим чем удвоенный эффективный радиус от центра КТ исчезающе мала.

\section{D. Влияние анизотропии KT на оптические свойства}

Рассмотрим влияние анизотропии КТ на энергии оптических переходов в КТ на примере КТ с фиксированным размером в направлении оси $z d_{z}=d=3 \mathrm{~nm}$ и $x=1$ в центре КТ. Это соответсвует значениям потенциального барьера $V_{\text {off }}^{\mathrm{e}}=0.81 \mathrm{eV}$ на границе КТ и жесткости по- 
тенциала в центре КТ $\kappa_{\mathrm{e}}^{z}=2 V_{\text {off }}^{\mathrm{e}} / a_{z}^{2}=0.72 \mathrm{eV} / \mathrm{nm}$, для электронов и $V_{\text {off }}^{\mathrm{h}}=0.27 \mathrm{eV}, \quad \kappa_{\mathrm{h}}^{z}=2 V_{\text {off }}^{\mathrm{h}} / a_{z}^{2}=24 \mathrm{eV} / \mathrm{nm}$ для дырок. На рис. 6 показаны потенциальные профили для электронов и дырок, энергии уровней и энергия оптического перехода для сферически-симметричной КТ (панель $(b)$ ), и аксиально анизотропных КТ с $d_{x}=6 \mathrm{~nm}$ для значения концентрации $\mathrm{Cd} x^{*}=0$ (панель $(c)$ ) и 0.2 (панель $(a))$ между точками в плоскости. Штрихпунтирные линии - соответствуют основному состоянию электронов и дырок, пунктирые - возбужденным, если таковые имеются. На панели $(a)$ сплошные линии соответствуют барьеру в плоскости структуры. Видно, что анизотропия размера КТ $\xi_{s}=d_{x}^{2} / d_{z}^{2}=4$ при одинаковом потенциальном барьере как вдоль оси роста, так и в плоскости структуры приводит как к уменьшению энергию оптического перехода на величину порядка $230 \mathrm{meV}$, так и к появлению локализованных возбужденных уровней электронов и дырок. Наличие локализованных возбужденных уровней позволяет резонансное оптическое возбуждение КТ. При дополнительном понижении потенциального барьера в плоскости, соответвующего $x^{*}=0.2$ (панель $(a)$ ), энергия перехода понижается еще на $20 \mathrm{meV}$, однако энергия локализации для основного состояния электронов и дырок в плоскости уменьшается, а возбужденные уровни делокализуются. При дальнейшем увеличении концентрации $\mathrm{Cd}$ между КТ до $x^{*}=0.4$ и соответсвующем понижении потенциальных барьеров в плоскости, локализация основных уровней энергии как дырки, так и электрона пропадает. При небольших энергиях локализации уровней, повышение температуры может приводить к тепловому выбросу носителей из КТ в плоскость структуры что может проявляться в уширении линии фотолюминесценции.

\section{4. Заключение}

Проведено рассмотрение влияния анизотропии формы и состава КТ на пороговые значения потенциальных барьеров, ниже которых локализация носителей отсутвует. При отрицательной энергии локализации вероятность обнаружить носитель между квантовыми точками в плоскости возрастает экспоненциально. Однако в области положительных, хотя и малых энергий локализации вероятность обнаружить электоны и дырки вне КТ крайне мала. Показано, что при исчезновении потенциального барьера в плоскости роста, энергии уровней стремятся к энергиям уровней размерного квантования в квантовой яме. Более того, меняется вид системы квантовых уровней от свойственного КТ к характерному для квантовой ямы. Значения пороговых значений потенциальных барьеров могут быть расчитаны, используя данные ПЭМ и микроанализа. Сравнение этих значений с величиной $k T$ ( $T$ - температура, при которой должен функционировать прибор, $k$ - постоянная Больцмана) может дать информацию о том, применим ли данный массив КТ для создания однофотонных излучателей, работающих, к примеру, при комнатной температуре, или же он оптимален для создания желтых лазеров, где требуется дополнительный сдвиг энергии в длинноволновую область, и для которых система уровней размерного квантования, типичная для КТ, не является необходимым условием.

\section{Список литературы}

[1] Single semiconductor quantum dots / Ed. P. Michler. Springer, Berlin (2009).

[2] M.D. Eisaman, J. Fan, A. Migdall, S.V. Polyakov. Rev. Sci. Instruments 82, 071101 (2011).

[3] N. Boto, P. Kok, D.S. Abrams, S.L. Braunstein, C.P. Williams, J.P. Dowling. Phys. Rev. Lett. 85, 2733 (2000).

[4] V. Giovannetti, S. Lloyd, L. Maccone. Science 306, 1330 (2004).

[5] C. Santori, D. Fattal, J. Vuckovic, G.S. Solomon, Y. Yamamoto. Nature 419, 594 (2002).

[6] Y.M. He, Y. He, Y.-J. Wei, D. Wu, M. Atature, C. Schneider, S. Höfling, M. Kamp, C.-Y. Lu, J.-W. Pan. Nature Nanotech. 8213 (2013).

[7] G. Sallen, A. Tribu, T. Aichele, R. Andrè, L. Besombes, C. Bougerol, S. Tatarenko, K. Kheng, J.Ph. Poizat. Phys. Rev. B 80, 085310 (2009).

[8] O. Fedorych, C. Kruse, A. Ruban, D. Hommel, G. Bacher, T. Kümmell. Appl. Phys. Lett. 100, 061114 (2012).

[9] W. Quitsch, T. Kimmell, A. Gust, C. Kruse, D. Hommel, G. Bacher. Appl. Phys. Lett. 105, 091102 (2014).

[10] A. A. Toropov, M.V. Rakhlin, K.G. Belyaev, S.V. Sorokin, G.V. Klimko, S.V. Gronin, I.V. Sedova, I.S. Mukhin, T.V. Shubina, S.V. Ivanov. J. Phys.: Conf. Ser. 917, 022001 (2017).

[11] S.V. Ivanov, A.A. Toropov, T.V. Shubina, S.V. Sorokin, A.V. Lebedev, I.V. Sedova, P.S. Kop'ev. J. Appl. Phys. 83, 3168 (1998).

[12] F. Gindele, U. Woggon, W. Langbein, J. M. Hvam, K. Leonardi, D. Hommel, H. Selke, Phys. Rev. B 60, 8773 (1999).

[13] A. Klochikhin, A. Reznitsky, B. Dal Don, H. Priller, H. Kalt, C. Klingshirn. Phys. Rev. B 69, 085308 (2004).

[14] M.V. Rakhlin, K.G. Belyaev, I.V. Sedova, S.V. Sorokin, S.V. Gronin, A.A. Usikova, A.A. Sitnikova, P.N. Brunkov, S.V. Ivanov, A.A. Toropov. Phys. Status Solidi C 13, 514 (2016).

[15] V. Türck, S. Rodt, O. Stier, R. Heitz, U.W. Pohl, R. Engelhardt, D. Bimberg. J. Lumin. 87-89, 337 (2000).

[16] L. Besombes, K. Kheng, L. Marsal, H. Mariette. Phys. Rev. B 63, 155307 (2001).

[17] M.V. Rakhlin, K.G. Belyaev, G.V. Klimko, I.S. Mukhin, D.A. Kirilenko, T.V. Shubina, S.V. Ivanov, A.A. Toropov. Sci. Rep. 8, 5229 (2018).

[18] N. Peranio, A. Rosenauer, D. Gerthsen, S.V. Sorokin, I.V. Sedova, S.V. Ivanov. Phys. Rev. B 61, 16015 (2000).

[19] D. Litvinov, A. Rosenauer, D. Gerthsen, N.N. Ledentsov. Phys. Rev. B 61, 16819 (2000).

[20] D. Litvinov, M. Schowalter, A. Rosenauer, B. Daniel, J. Fallert, W. Löffler, H. Kalt, M. Hetterich. Phys. Status Solidi A 205, 2892 (2008).

[21] С.В. Сорокин, И.В. Седова, К.Г. Беляев, М.В. Рахлин, М.А. Яговкина, А.А. Торопов, С.В. Иванов. Письма в ЖТФ 6, 94 (2018). 
[22] T.V. Shubina, A.V. Rodina, M.A. Semina, A.A. Golovatenko, A.A. Toropov, M.V. Rakhlin, I.V. Sedova, S.V. Sorokin, S.V. Gronin, A.A. Sitnikova, D.I. Kuritsyn, S.M. Sergeev, Z.F. Krasil'nik, S.V. Ivanov. Phys. Status Solidi B 253, 1485 (2016).

[23] A.A. Golovatenko, M.A. Semina, A.V. Rodina, T.V. Shubina. Acta Phys. Polon. A 129, 107 (2016).

[24] Т.В. Шубина, К.Г. Беляев, М.А. Семина, А.В. Родина, А.А. Головатенко, А.А. Торопов, С.В. Сорокин, И.В. Седова, В.Ю. Давыдов, А.Н. Смирнов, П.С. Копьев, С.В. Иванов. ФТТ 58, 2175 (2016).

[25] M.A. Semina, A.A. Golovatenko, A.V. Rodina. Phys. Rev. B 93, 045409 (2016).

[26] А.А. Головатенко М.А. Семина, А.В. Родина, Т.В. Шубина, ФTT 8, 1499 (2018).

[27] S. Adachi. Properties of Semiconductor Alloys: Group-IV, III-V and II-VI Semiconductors. Chippenham. John Wiley and Sons Ltd (2009).

[28] J.M. Luttinger. Phys. Rev. 102, 1030 (1956).

[29] B.L. Gel'mont, M.I. D’yakonov. Sov. Phys. Semicond. 5, 905 (1971).

[30] Al.L. Efros, M. Rosen, M. Kuno, M. Nirmal, D.J. Norris, M. Bawendi. Phys. Rev. B 54, 4843 (1996).

Редактор Т.Н. Василевская 